\title{
DYNAMIC PROGRAMMING FOR THE STOCHASTIC NAVIER-STOKES EQUATIONS *
}

\author{
Giuseppe da Prato ${ }^{1}$ And Arnaud Debussche ${ }^{2}$
}

\begin{abstract}
We solve an optimal cost problem for a stochastic Navier-Stokes equation in space dimension 2 by proving existence and uniqueness of a smooth solution of the corresponding HamiltonJacobi-Bellman equation.
\end{abstract}

Mathematics Subject Classification. 35B37, 60H15, 76D05, 93E20.

Received: November 18, 1999.

\section{INTRODUCTION}

In this article, we study the dynamic programming approach for the control of turbulent flows. This control problem has many industrial and engineering applications. In the context of aeronautics, the aim is to reduce turbulence and this work is motivated by this application. In other areas, one wants to maximize turbulence, this is the case in combustion where it is desirable to optimize the mixing of the components.

This problem has been the object of many articles. In [1], optimality conditions are derived and a numerical algorithm is proposed. Then, in $[7,18]$ this approach is further developed and tested numerically. Robust control theory for this problem has also been addressed in [2] and in [3].

Also in $[11,15,16]$, the existence of an optimal control has been proved thanks to the Pontryagin maximum principle and it is shown that the minimum value function is a viscosity solution (see $[14,17]$ ) to the HamiltonJacobi-Bellman (HJB) equation associated to the problem. Unfortunately, viscosity solutions are not smooth enough to fully justify the dynamic programming approach.

In this work, we follow a strategy proposed in $[4,5]$ to get smooth solutions to HJB equations. We consider a model problem where the flow is two dimensional, governed by the stochastic Navier-Stokes equations and the turbulence is to be minimized thanks to a distributed control. More realistic problems, with boundary controls for instance, will be considered in future works. The unknowns are the velocity $U(\xi, t)=\left(U_{1}(\xi, t), U_{2}(\xi, t)\right)$ and

\footnotetext{
Keywords and phrases. Stochastic Navier-Stokes equations, dynamic programming, optimal control, Hamilton-Jacobi-Bellmann equations.

* Dedicated to Roger Temam for his 60th birthday

1 Scuola Normale Superiore di Pisa, Piazza dei Cavalieri 7, 56126 Pisa, Italy.

2 CNRS et Université de Paris-Sud, 91405 Orsay Cedex, France. e-mail: arnaud.debussche@math.u-psud.fr
} 
the pressure $p(\xi, t)$ defined for $\xi$ in a bounded open subset $D$ of $\mathbb{R}^{2}$ and satisfy

$$
\left\{\begin{array}{l}
\frac{\partial U}{\partial t}+(U . \nabla) U+\nabla p=\nu \Delta U+z+\dot{\eta}, \text { in } D \\
\operatorname{div} U=0, \text { in } D \\
U=0, \text { on } \partial D \\
\left.U\right|_{t=0}=u_{0}, \text { in } D .
\end{array}\right.
$$

The control has been denoted by $z=z(\xi, t)$, it is a random variable with values in the space of square integrable functions, $\nu$ is the kinematic viscosity and $\dot{\eta}$ is a random term of white noise type. Since, it does not play a particular role in this work we will, with no loss of generality, take $\nu=1$. Intuitively, the amount of turbulence in a flow can be measured by the time averaged enstrophy and it seems natural to try to minimize the following cost functional:

$$
J(z)=\mathbb{E} \int_{0}^{T} \int_{D}\left(|\operatorname{rot} U(\xi, t)|^{2}+|z(\xi, t)|^{2}\right) \mathrm{d} \xi \mathrm{d} t+\int_{D}|U(\xi, T)|^{2} \mathrm{~d} \xi
$$

The last term is not essential and could be omitted.

The HJB equation associated to this problem is a second order partial differential equation whose unknown depends on $t$ and on a variable $x$ in $H$, the Hilbert space of square integrable divergence free functions. The linear part of which is the Kolmogorov equation associated to the uncontrolled Navier-Stokes equations and whose solution is given by the transition semigroup. The nonlinear part of the HJB equation involves the derivative of the unknown, it is quadratic if the control is allowed to be any square integrable process and Lipschitz if we impose a boundedness condition on the control.

Hence, a way to find a smooth solution to the HJB equation is to write it in a mild form thanks to the transition semigroup and to use a fixed point argument. This requires some smoothing properties of the transition semigroup. This methodology has been successfully implemented in the case of equations with Lipschitz nonlinearities [12,13] and of reaction-diffusion equations [6]. For the control of the stochastic Burgers equation, smoothing properties of the transition semigroup have recently been proved in [8]. However it is shown that the transition semigroup maps bounded continuous functions to smooth functions with exponential growth and a fixed point strategy does not seem to apply. This difficulty has been overcomed by using a compactness argument after delicate a priori estimates are proved. In this way, the dynamic programming approach has been fully justified in the case of bounded control in [9].

The Navier-Stokes equations contain additional difficulties. First, it is not possible to have a noise term $\dot{\eta}$ such that $J(z)$ is well defined and such that, at the same time, the transition semigroup is smoothing. Even, in the case of the stochastic heat equation in space dimension 2, this is not possible. A smoothing effect of the transition semigroup is possible only if we consider functions defined on a smaller space than $H$. This forces us to assume that the control has some regularity properties. Thus we slightly change the problem by assuming that the control is subjected to the action of a linear operator $B$ which maps $H$ into a smaller Hilbert space in which the transition semigroup can have a smoothing effect. In other words, we replace $z$ by $B z$ in (1.1). We think that this assumption is not restrictive since in practise controls are smooth functions.

Then one could use the same type of arguments as in [8] to prove a smoothing property but another difficulty appears. The introduced exponential factor is very large and we are not able to apply the method of [9]. The trick we use in this paper is to change the unknown of the HJB equation by multiplying it by an exponential factor. In this way, the HJB equation is transformed into a similar equation but now the linear part corresponds to a Feynmann-Kac semigroup with a potential. Thanks to this potential, we are able to prove nice smoothing properties of this Feynmann-Kac semigroup. It maps bounded functions to smooth functions with polynomial growth. The fixed point argument does not seem to apply and we use the same type of argument as in [9] to find a smooth solution to the transformed HJB equation. We deduce the existence of a smooth solution $u$ to the HJB equation. Since we are able to justify the formal identity which gives a relationship between $J(z)$, for 
any $z$, and $u$ and since we can prove existence of a unique solution to the closed loop equation, we can use this solution to construct a unique optimal control. In this way, we fully justify the dynamic programming approach in our case.

To our knowledge, these arguments are new. They are general and can be used for other control problems: boundary control, other partial differential equations, ... Also, similar ideas will be used in a forthcoming work to study the Dirichlet form associated to the stochastic Navier-Stokes equations in space dimension 2.

\section{Notations AND MAin RESUlts}

Let $D \subset \mathbb{R}^{2}$ be a bounded open set of regular boundary $\partial D$ in $\mathbb{R}^{2}$ and let $L^{2}(D)$ be the Hilbert space of all real valued square Lebesgue integrable functions on $D$. We denote by $H^{k}(D), k \in \mathbb{N}$, the usual Sobolev spaces, and by $H_{0}^{1}(D)$ the space of all functions in $H^{1}(D)$ whose trace on $\partial D$ vanishes. Classicaly, we introduce the Hilbert spaces

$$
\begin{gathered}
H=\left\{u \in\left(L^{2}(D)\right)^{2}: \operatorname{div} u=0 \text { in } D, u \cdot n=0 \text { in } \partial D\right\}, \\
V=\left\{u \in\left(H_{0}^{1}(D)\right)^{2}: \operatorname{div} u=0 \text { in } D\right\},
\end{gathered}
$$

where $n$ denotes the outward unit normal vector on $\partial D ; H$ (resp. $V$ ) is endowed with the inner product and norm of $\left(L^{2}(D)\right)^{2},\left(\operatorname{resp} .\left(H_{0}^{1}(D)\right)^{2}\right)$ denoted by $(\cdot, \cdot)$ and $|\cdot|(\operatorname{resp} .((\cdot, \cdot))$ and $\|\cdot\|)$.

We also introduce the unbounded self-adjoint operator

$$
A=P \Delta, \quad D(A)=\left(H^{2}(D)\right)^{2} \cap V,
$$

where $P$ is the orthogonal projector of $\left(L^{2}(D)\right)^{2}$ onto $H$.

The operator $A$ has a compact resolvent and possesses a basis $\left(e_{k}\right)$ of eigenvectors associated to the eigenvalues:

$$
0>-\lambda_{1} \geq-\lambda_{2} \geq \ldots-\lambda_{k} \rightarrow-\infty
$$

The eigenprojector $P_{n}$ onto $\operatorname{Sp}\left(e_{1}, \ldots, e_{n}\right)$ will be used to define an approximation of the Navier-Stokes equations.

Also we will use the spaces $D\left((-A)^{s}\right)$ which are defined for any $s \in \mathbb{R}$. We set

$$
|\cdot|_{s}=\left|(-A)^{s} \cdot\right|, \quad s \in \mathbb{R} .
$$

We have

$$
D\left((-A)^{0}\right)=H, \quad D\left((-A)^{1 / 2}\right)=V .
$$

Furthermore, if $s_{1}<s_{2}$, the embedding $D\left((-A)^{s_{2}}\right) \subset D\left((-A)^{s_{1}}\right)$ is compact.

The space (resp. time) variable will be denoted by $\xi \in D$ (resp. $t \geq 0$ ) and $\nabla_{\xi}$ (resp. $D_{t}$ ) denotes the derivative with respect to $\xi$ (resp. $t$ ).

The bilinear form $b$ is defined by

$$
b(x, y)=P\left(\left(x \cdot \nabla_{\xi}\right) y\right), \quad x, y \in V .
$$


It can be extended to more general $x, y$ in various ways. When $x=y$, we set

$$
b(x)=b(x, x), \quad x \in V .
$$

Among others, $b$ has the following fundamental properties:

$$
\begin{gathered}
(b(x, y), y)=0, \\
(b(x, y), z)=-(b(x, z), y), \\
|(b(x, y), z)| \leq\left. c_{1}(\sigma)|x|_{\sigma}\|y\||| z\right|_{\frac{1}{2}-\sigma},
\end{gathered}
$$

for $x, y, z \in V$ and $\sigma \in(0,1 / 2)$, the constant $c_{1}(\sigma)$ depending on $\sigma$ and on $D$ (see [19]).

The noise term is described by a stochastic basis $\left(\Omega, \mathcal{F}, \mathbb{P},\left(\mathcal{F}_{t}\right)_{t \geq 0}\right)$, a cylindrical Wiener process on $H$, $(W(t))_{t \geq 0}$, associated to this basis, and a covariance operator $Q$ which is symmetric and positive.

The control $z$ is chosen in the space

$$
\mathcal{M}_{R}=\left\{z \in L_{W}^{2}(\Omega \times[0, T] ; H),|z| \leq R\right\},
$$

where the subscript $W$ means that we consider adapted processes. The number $R$ is fixed throughout the paper. This control is subject to the action of a linear operator $B \in \mathcal{L}(H)$. Now, we can rewrite the controlled Navier-Stokes equations in the abstract form

$$
\left\{\begin{array}{l}
\mathrm{d} X(t)=(A X(t)+b(X(t))+B z(t)) \mathrm{d} t+Q^{1 / 2} \mathrm{~d} W(t) \\
X(0)=x \in H .
\end{array}\right.
$$

Using classical arguments, it is easy to prove that (2.4) has a unique solution $X$. Equation (2.4) is associated to the cost functional

$$
J(z)=\mathbb{E}\left(\int_{0}^{T}\left|\operatorname{rot}_{\xi} X(s)\right|^{2}+\frac{1}{2}|z(s)|^{2} \mathrm{~d} s+|X(T)|^{2}\right),
$$

where $T>0$ is also a fixed number and

$$
\operatorname{rot}_{\xi} X=\frac{\partial X_{1}}{\partial \xi_{2}}-\frac{\partial X_{2}}{\partial \xi_{1}}
$$

is the rotational of $X=\left(X_{1}, X_{2}\right)$.

Our aim is to find $z^{*} \in \mathcal{M}_{R}$ which minimize $J(z)$ :

$$
J\left(z^{*}\right)=\min _{z \in \mathcal{M}_{R}} J(z)
$$

This control problem is a model problem for the control of turbulence in a viscous and incompressible fluid. Using the Itô formula it is easy to see that $J$ has only infinite values unless

$$
\operatorname{Tr} Q<+\infty,
$$


thus we assume from now on that this condition is satisfied.

We follow the dynamic programming approach to solve this problem. Let the Hamiltonian $F$ be defined on $H$ by

$$
F(p)=\left\{\begin{array}{l}
\frac{1}{2}\left|B^{*} p\right|^{2}, \text { if }\left|B^{*} p\right| \leq R \\
\left|B^{*} p\right| R-\frac{1}{2} R^{2}, \text { if }\left|B^{*} p\right| \geq R
\end{array}\right.
$$

The Hamilton-Jacobi-Bellman equation associated with our control problem is

$$
\left\{\begin{array}{l}
D_{t} u=\frac{1}{2} \operatorname{Tr}\left[Q u_{x x}\right]+\left(A x+b(x), u_{x}\right)-F\left(u_{x}\right)+g(x), x \in H, t \in[0, T], \\
u(0, x)=|x|^{2}, x \in H,
\end{array}\right.
$$

where the subscript $x$ means differentiation with respect to the variable $x \in H$ and $g(x)=\left|\operatorname{rot}_{\xi} x\right|^{2}$. If we are able to find a smooth solution $u$ of (2.8), then the optimal control is given by the formula

$$
z^{*}(t)=-D_{p} F\left(u_{x}\left(T-t, X^{*}(t)\right)\right),
$$

where the optimal state $X^{*}$ is the solution of the closed loop equation

$$
\left\{\begin{array}{l}
\mathrm{d} X^{*}(t)=\left(A X^{*}(t)+b\left(X^{*}(t)\right)-D_{p} F\left(u_{x}\left(T-t, X^{*}(t)\right)\right)\right) \mathrm{d} t+Q^{1 / 2} \mathrm{~d} W(t) \\
X(0)=x \in H
\end{array}\right.
$$

A way to find a smooth solution $u$ of (2.8), is to introduce the transition semigroup $\left(P_{t}\right)_{t \geq 0}$ associated to the uncontrolled Navier-Stokes equations. It is defined by

$$
P_{t} \varphi(x)=\mathbb{E}[\varphi(Y(t, x))]
$$

where $\varphi: H \rightarrow \mathbb{R}$ is a Borel function and $Y$ satisfies

$$
\left\{\begin{array}{l}
\mathrm{d} Y(t)=(A Y(t)+b(Y(t))) \mathrm{d} t+Q^{1 / 2} \mathrm{~d} W(t) \\
Y(0)=x \in H
\end{array}\right.
$$

Then we write $(2.8)$ in the mild form

$$
u(t, \cdot)=P_{t} \varphi(\cdot)+\int_{0}^{t} P_{t-s} F\left(u_{x}(s, \cdot)\right) \mathrm{d} s+\int_{0}^{t} P_{t-s} g(\cdot) \mathrm{d} s,
$$

where $\varphi(x)=|x|^{2}$. Due to the loss of one derivative in the second term of the right hand side, solving (2.12) requires some smoothing properties of the transition semigroup. We need to know that $P_{t}$ maps continuous into differentiable functions. For equations with Lipschitz nonlinearities or in the case of reaction-diffusion equations, such properties hold (provided $Q$ is suitably chosen), and a fixed point strategy can be used to find a smooth $u$ satisfying $(2.11)$ (see $[4-6,12,13]$ ). When the Burgers equation is considered a smoothing effect for $\left(P_{t}\right)$ can still be proved but now a factor of exponential growth appears and it seems that a fixed point strategy cannot be used. Using the fact that the introduced exponential growth is arbitrarily small, this problem has been overcomed in [9]. A compactness argument has been used to find a solution $u$ and the control problem has been solved.

In the case of the Navier-Stokes equations considered here, two difficulties appear. First, the transition semigroup does not map continuous on differentiable functions on $H$. This is due to assumption (2.6) and even 
in the linear case, i.e. if we take $b=0$, the transition semigroup does not have such a smoothing property. However, it can be seen that $\left(P_{t}\right)$ has a weaker smoothing property. It can be proved that if $\varphi$ is continuous, $P_{t} \varphi$ is differentiable at any point $x$, in a smaller space $D\left((-A)^{\gamma}\right)$ for some $\gamma>0$, along directions in this same space. This is true under the assumption that $Q$ is not too degenerate:

$$
\left|Q^{-1 / 2} x\right| \leq c_{Q}\left|(-A)^{\frac{1}{2}+\gamma_{1}} x\right| \text {, for any } x \in D\left((-A)^{\frac{1}{2}+\gamma_{1}}\right),
$$

with $\frac{1}{2}>\gamma_{1}>0$. The second difficulty is that, again, exponential growth are introduced but here they are not arbitrarily small and, apparently, the technique in [9] fails.

We then introduce a new ingredient which consists in the following change of unknown:

$$
v(t, x)=e^{-K|x|^{2}} u(t, x), t \geq 0, x \in D .
$$

This transforms $(2.8)$ in

$$
\left\{\begin{aligned}
D_{t} v= & \frac{1}{2} \operatorname{Tr}\left[Q v_{x x}\right]+\left(A x+b(x), v_{x}\right) \\
& -2 K\|x\|^{2} v+\widetilde{F}\left(x, v, v_{x}\right)+\widetilde{g}(x), x \in H, t \in[0, T], \\
v(0, x)= & \widetilde{\varphi}(x), x \in H
\end{aligned}\right.
$$

where

$$
\widetilde{F}\left(x, v, v_{x}\right)=2 K\left(Q x, v_{x}\right)+\left(2 K^{2}\left|Q^{1 / 2} x\right|^{2}+2 K \operatorname{Tr} Q\right) v-e^{-K|x|^{2}} F\left(e^{K|x|^{2}}\left(v_{x}+2 K v x\right)\right),
$$

and

$$
\widetilde{g}(x)=e^{-K|x|^{2}} g(x), \quad \widetilde{\varphi}(x)=e^{-K|x|^{2}}|x|^{2} .
$$

We associate the Feynman-Kac semigroup

$$
R_{t} \varphi(x)=\mathbb{E}\left(e^{-2 K \int_{0}^{t}\|Y(s, x)\|^{2} \mathrm{~d} s} \varphi(Y(t, x))\right),
$$

for $\varphi: H \rightarrow \mathbb{R}$ Borel, $x \in H$ and with $Y$ solution of $(2.11)$. Then $\zeta(t, x)=R_{t} \varphi(x)$ is formally the solution of the linear equation

$$
\left\{\begin{array}{l}
D_{t} \zeta \quad=\frac{1}{2} \operatorname{Tr}\left[Q \zeta_{x x}\right]+\left(A x+b(x), \zeta_{x}\right)-2 K\|x\|^{2} \zeta, \quad x \in H, t \in[0, T] \\
\zeta(0, x)=\widetilde{\varphi}(x), x \in H
\end{array}\right.
$$

and (2.15) can be rewritten in the mild form

$$
v(t, \cdot)=R_{t} \widetilde{\varphi}(\cdot)+\int_{0}^{t} R_{t-s} \widetilde{F}\left(\cdot, v(s, \cdot), v_{x}(s, \cdot)\right) \mathrm{d} s+\int_{0}^{t} R_{t-s} \widetilde{g}(\cdot) \mathrm{d} s .
$$

Due to the presence of the exponential inside the expectation, $\left(R_{t}\right)$ has nicer properties than $\left(P_{t}\right)$. It has a similar type of smoothing property in a smaller space than $H$ under the assumption (2.13) and, instead of introducing exponential growth, it introduces only polynomial growth.

We now define spaces of functions on $D\left((-A)^{\gamma}\right), \gamma \geq 0$, having polynomial growth. For $\gamma=0, k \in \mathbb{N}, l \in \mathbb{N}$, and $\alpha \in[0,1]$ we define

$$
C^{0, k, l+\alpha}=\left\{\psi: H \rightarrow \mathbb{R}: \psi \text { is } l \text { times differentiable and }|\psi|_{0, k, l+\alpha}<\infty\right\},
$$


where $\left({ }^{1}\right)$

$$
|\psi|_{0, k, l+\alpha}=\sup _{x \in H}(1+|x|)^{-k}|\psi(x)|+\sup _{r>0}(1+r)^{-k} \sup _{|x| \leq r,|y| \leq r} \frac{\left|D^{l} \psi(x)-D^{l} \psi(y)\right|_{\mathcal{L}\left(H^{l}, \mathbb{R}\right)}}{|x-y|^{\alpha}}
$$

For $\gamma>0$ we set

$$
C^{\gamma, k, l+\alpha}=\left\{\varphi: D\left((-A)^{\gamma}\right) \rightarrow \mathbb{R}: \varphi\left((-A)^{-\gamma} \cdot\right) \in C^{0, k, l+\alpha}\right\}
$$

and

$$
|\varphi|_{\gamma, k, l+\alpha}=\left|\varphi\left((-A)^{-\gamma} \cdot\right)\right|_{0, k, l+\alpha}
$$

It is easily seen that

$$
|\varphi|_{\gamma, k, 1}=\sup _{x \in D\left((-A)^{\gamma}\right)}\left(1+|x|_{\gamma}\right)^{-k}|\varphi(x)|+\sup _{x \in D\left((-A)^{\gamma}\right)}\left(1+|x|_{\gamma}\right)^{-k} \sup _{h \in D\left((-A)^{\gamma}\right)} \frac{(D \varphi(x), h)}{|h|_{\gamma}}
$$

and

$$
|\varphi|_{\gamma, k, 2}=\sup _{x \in D\left((-A)^{\gamma}\right)}\left(1+|x|_{\gamma}\right)^{-k}|\varphi(x)|+\sup _{x \in D\left((-A)^{\gamma}\right)}\left(1+|x|_{\gamma}\right)^{-k} \sup _{h \in D\left((-A)^{\gamma}\right)} \frac{\left(D^{2} \varphi(x) h, h\right)}{|h|_{\gamma}^{2}}
$$

Also for $\lambda \in[0,1], C^{\gamma, k, l+\alpha}$ is the interpolation space of $C^{\gamma, k_{1}, l_{1}+\alpha_{1}}$ and $C^{\gamma, k_{2}, l_{2}+\alpha_{2}}$ with

$$
(k, l+\alpha)=\lambda\left(k_{1}, l_{1}+\alpha_{1}\right)+(1-\lambda)\left(k_{2}, l_{2}+\alpha_{2}\right)
$$

We will prove that if $\gamma>\gamma_{1}, R_{t}$ maps $C^{\gamma, k, \beta}$ into $C^{\gamma, k+2 \alpha, \alpha+\beta}$ whenever $1 \geq \beta \geq 0$ and $0 \leq \alpha+\beta \leq 2$. Due to the loss in the exponent describing the polynomial growth it is again impossible to solve (2.17) by a fixed point argument. Instead we construct a solution by a compactness method.

Due to the fact that we can only prove smoothing effects in $D\left((-A)^{\gamma}\right)$ with $\gamma>\gamma_{1}$, the differential of the solution will not belong to $H$ but to $D\left((-A)^{\gamma}\right)$ for some $\gamma>\gamma_{1}$. Thus we need a further assumption on $B$, namely

$$
|B x| \leq c_{B}\left|(-A)^{-\widetilde{\gamma_{1}}} x\right|, \forall x \in D\left((-A)^{-\widetilde{\gamma_{1}}}\right),
$$

for some $\frac{1}{2}>\widetilde{\gamma_{1}}>\gamma_{1}$.

In this way we prove in Sections 3 and 4 the following result.

Theorem 2.1. Assume that (2.6), (2.13) hold and that $K \geq K_{1}\left(\gamma_{1}, \tilde{\gamma}_{1}\right)$. Then there exists a function $v \in$ $C\left([0, T] ; C^{\gamma, d, 2}\right)$ for any $\gamma \in\left(\gamma_{1}, \tilde{\gamma_{1}}\right)$, with $d \geq 0$ depending on $\gamma, \gamma_{1}$, and $\tilde{\gamma_{1}}$, such that $v$ satisfies (2.17), i.e. which is a mild solution to the transformed equation (2.16).

\footnotetext{
${ }^{1}$ The expression below needs an obvious modification if $\alpha=0$.
} 
Now setting $u(t, x)=e^{K|x|^{2}} v(t, x)$, it can be shown that $u$ is a mild solution to (2.8). It is thereby continuous in time with value in a space of $C^{2}$ functions on $D\left((-A)^{\gamma}\right)$ with exponential growth. It follows that $u_{x}(t, \cdot)$ is locally Lipschitz from $D\left((-A)^{\gamma}\right)$ to $D\left((-A)^{-\gamma}\right)$ for $\gamma \in\left(\gamma_{1}, \tilde{\gamma_{1}}\right)$. (This can be obtained from (4.6) below for instance). More precisely, for any $r \geq 0$, there exists $L(r)$ such that for $x, y \in D\left((-A)^{\gamma}\right), t \in[0, T]$ :

$$
|D u(t, x)-D u(t, y)|_{-\gamma} \leq L(r)|x-y|_{\gamma},
$$

if $|x|_{\gamma},|y|_{\gamma} \leq r$.

Also, taking the limit in (2.24) below and using similar arguments as in [8], we deduce that for any $z \in \mathcal{M}_{R}$ we have

$$
J(z)=u(T, x)+\frac{1}{2} \mathbb{E}\left(\int_{0}^{T}\left|z(s)+B^{*} u_{x}(T-s, X(s, x))\right|^{2}-\chi\left(\left|B^{*} u_{x}(T-s, X(s, x))\right|-R\right) \mathrm{d} t\right),
$$

where $\chi(a)=a^{2}$ for $a \geq 0$ and $\chi(a)=0$ for $a \leq 0$.

Also, thanks to (2.19) and since $\gamma<1 / 2$, it can be checked that the closed loop equation (2.10) has a unique solution $X^{*}$. Hence, there exists a unique optimal control $z^{*}$ which is given by (2.9). Therefore we can state our second main result which justifies completely the dynamic programming approach for our control problem.

Theorem 2.2. Assume that (2.6), (2.13) hold. Then, for any control $z \in \mathcal{M}_{R}$, the fundamental identity (2.20) holds. Moreover the closed loop equation (2.10) has a unique solution $X^{*}$ and there exists a unique optimal control $z^{*}$ which is given by (2.9).

We now describe the approximation scheme used to prove Theorem 2.1.

The approximated solution to (2.17) will be obtained thanks to a standard Galerkin approximation. For $m \in \mathbb{N}$, we consider the control problem in finite dimension which consists in minimizing

$$
J_{m}\left(z_{m}\right)=\mathbb{E}\left(\int_{0}^{T}\left|\operatorname{rot}_{\xi} X_{m}(s)\right|^{2}+\frac{1}{2}\left|z_{m}(s)\right|^{2} \mathrm{~d} s+\left|X_{m}(T)\right|^{2}\right),
$$

for $z_{m} \in \mathcal{M}_{R} \cap L_{W}^{2}\left(\Omega \times[0, T] ; P_{m} H\right)$, where

$$
\left\{\begin{array}{l}
\mathrm{d} X_{m}(t)=\left(A X_{m}(t)+b_{m}\left(X_{m}(t)\right)+B_{m} z_{m}(t)\right) \mathrm{d} t+Q_{m}^{1 / 2} \mathrm{~d} W(t) \\
X_{m}(0)=x \in P_{m} H
\end{array}\right.
$$

with $b_{m}(x)=P_{m} b\left(P_{m} x\right), B_{m}=P_{m} B P_{m}, Q_{m}=P_{m} Q P_{m}$. Then we consider the following equation for $v^{m}$,

$$
\left\{\begin{aligned}
D_{t} v^{m}= & \frac{1}{2} \operatorname{Tr}\left[Q_{m} v_{x x}^{m}\right]+\left(A x+b_{m}(x), v_{x}^{m}\right) \\
& -2 K\|x\|^{2} v^{m}+\widetilde{F}_{m}\left(x, v^{m}, v_{x}^{m}\right)+\widetilde{g}(x), \quad x \in P_{m} H, t \in[0, T], \\
v^{m}(0, x)= & \widetilde{\varphi}(x), x \in P_{m} H,
\end{aligned}\right.
$$

where,

$$
\widetilde{F}_{m}\left(x, v^{m}, v_{x}^{m}\right)=2 K\left(Q_{m} x, v_{x}^{m}\right)+\left(2 K^{2}\left|Q_{m}^{1 / 2} x\right|^{2}+2 K \operatorname{Tr} Q_{m}\right) v-e^{-K|x|^{2}} F\left(e^{K|x|^{2}}\left(v_{x}^{m}+2 K v^{m} x\right)\right) .
$$

We associate the Feynman-Kac semigroup $\left(R_{t}^{m}\right)$

$$
R_{t}^{m} \varphi(x)=\mathbb{E}\left(e^{-2 K \int_{0}^{t}\left\|Y_{m}(s, x)\right\|^{2} \mathrm{~d} s} \varphi\left(Y_{m}(t, x)\right)\right)
$$


for $\varphi: P_{m} H \rightarrow \mathbb{R}$ Borel, $x \in P_{m} H . Y_{m}$ solves the equation

$$
\left\{\begin{array}{l}
D_{t} Y_{m}=\left(A Y_{m}+b_{m}\left(Y_{m}\right)\right) \mathrm{d} t+Q_{m}^{1 / 2} \mathrm{~d} W \\
Y_{m}(0)=x
\end{array}\right.
$$

and (2.21) has the following mild form

$$
v^{m}(t, \cdot)=R_{t}^{m} \widetilde{\varphi}(\cdot)+\int_{0}^{t} R_{t-s}^{m} \widetilde{F}_{m}\left(\cdot, v^{m}(s, \cdot), v_{x}^{m}(s, \cdot)\right) \mathrm{d} s+\int_{0}^{t} R_{t-s}^{m} \widetilde{g}(\cdot) \mathrm{d} s .
$$

It is not difficult to prove that there exists a smooth function $v^{m}$ satisfying (2.21) and (2.23). Indeed this is a Hamilton-Jacobi-Bellman equation in finite dimension with smooth coefficients. The only slight difficulty is due to the quadratic term $b_{m}$ but this may be overcomed very easily by a truncation argument for instance.

Once $v^{m}$ is obtained, it is easy to write the equation for $u^{m}(t, x)=e^{K|x|^{2}} v^{m}(t, x)$ and a classical computation based on the Itô formula proves that for any $z_{m} \in \mathcal{M}_{R} \cap L_{W}^{2}\left(\Omega \times[0, T] ; P_{m} H\right)$ :

$$
J_{m}\left(z_{m}\right)=u^{m}(T, x)+\frac{1}{2} \mathbb{E}\left(\int_{0}^{T}\left|z_{m}(s)+B^{*} u_{x}^{m}\left(T-s, X_{m}(s, x)\right)\right|^{2}-\chi\left(\left|B^{*} u_{x}^{m}\left(T-s, X_{m}(s, x)\right)\right|-R\right) \mathrm{d} t\right)
$$

so that the optimal control is obtained by taking

$$
z_{m}^{*}(t)=-D_{p} F\left(u_{x}^{m}\left(T-t, X_{m}^{*}(t, x)\right)\right.
$$

where $X_{m}^{*}$ solves the closed loop equation

$$
\left\{\begin{array}{l}
\mathrm{d} X_{m}^{*}(t)=\left(A X_{m}^{*}(t)+b_{m}\left(X_{m}^{*}(t)\right)-D_{p} F\left(u_{x}^{m}\left(T-t, X_{m}^{*}(t)\right)\right)\right) \mathrm{d} t+Q_{m}^{1 / 2} \mathrm{~d} W(t) \\
X_{m}^{*}(0)=x \in H
\end{array}\right.
$$

It is easy to prove existence and uniqueness of $X_{m}^{*}$.

We will use the differential of $Y_{m}(t, x)$ with respect to the initial data. For $h \in P_{m} H$ we set $\eta_{m}^{h, x}(t)=$ $\left(D Y_{m}(t, x), h\right) ; \eta_{m}^{h, x}$ is the solution of

$$
\left\{\begin{array}{l}
D_{t} \eta_{m}^{h, x}=A \eta_{m}^{h, x}+b_{m}\left(Y_{m}(t, x), \eta_{m}^{h, x}\right)+b_{m}\left(\eta_{m}^{h, x}, Y_{m}(t, x)\right) \\
\eta_{m}^{h, x}(0)=h
\end{array}\right.
$$

The second differential in the directions $h, k \in P_{m} H$ is denoted by $\zeta_{m}^{h, k, x}(t)=\left(D^{2} Y_{m}(t, x) h, k\right) ; \zeta_{m}^{h, h, x}$ is the solution of

$$
\left\{\begin{aligned}
D_{t} \zeta_{m}^{h, k, x}= & A \zeta_{m}^{h, k, x}+b_{m}\left(Y_{m}(t, x), \zeta_{m}^{h, k, x}\right)+b_{m}\left(\zeta_{m}^{h, k, x}, Y_{m}(t, x)\right) \\
& +2 b_{m}\left(\eta_{m}^{h, x}(t), \eta_{m}^{h, x}(t)\right) \\
\zeta_{m}^{h, k, x}(0)= & 0
\end{aligned}\right.
$$

Often we will write $\eta_{m}^{h}$ and $\zeta_{m}^{h}$ or even $\eta_{m}$ or $\zeta_{m}$ instead of $\eta_{m}^{h, x}$ and $\zeta_{m}^{h, h, x}$.

In all the article, we use $c_{i}$ or $C_{i}$ to denote constants that may depend on $A, Q, b, T, B$ or $D$. These will never depend on $m$. When they depend on another parameter, it will be explicitly precised. 


\section{Smoothing properties of the Feynman-Kac semigroup}

Following [10], we have the following generalization of the Bismut-Elworthy formula which expresses the differential of $R_{t}^{m} \varphi$ :

$$
\begin{aligned}
\left(D R_{t}^{m} \varphi(x), h\right)= & \mathbb{E}\left(e ^ { - 2 K \int _ { 0 } ^ { t } \| Y _ { m } ( s , x ) \| ^ { 2 } \mathrm { d } s } \varphi ( Y _ { m } ( t , x ) ) \left[\frac{1}{t} \int_{0}^{t}\left(Q_{m}^{-1 / 2} \eta_{m}^{h, x}(s), \mathrm{d} W(s)\right)\right.\right. \\
& \left.\left.+4 K \int_{0}^{t}\left(1-\frac{s}{t}\right)\left(\left(\eta_{m}^{h, x}(s), Y_{m}(s, x)\right)\right) \mathrm{d} s\right]\right),
\end{aligned}
$$

for $\varphi \in C_{b}\left(P_{m} H\right), x \in P_{m} H, h \in P_{m} H$ and $t \in[0, T]$. Moreover, by differentiating (3.1) we obtain

$$
\begin{aligned}
\left(D^{2} R_{t}^{m} \varphi(x) h, h\right)= & \mathbb{E}\left(e ^ { - 2 K \int _ { 0 } ^ { t } \| Y _ { m } ( s , x ) \| ^ { 2 } \mathrm { d } s } \varphi ( Y _ { m } ( t , x ) ) \left[\frac{1}{t} \int_{0}^{t}\left(Q_{m}^{-1 / 2} \zeta_{m}^{h, x}(s), \mathrm{d} W(s)\right)\right.\right. \\
+ & \left.4 K \int_{0}^{t}\left(1-\frac{s}{t}\right)\left\{\left(\left(\zeta_{m}^{h, x}(s), Y_{m}(s, x)\right)\right)+\left\|\eta_{m}^{h, x}(s)\right\|^{2}\right\} \mathrm{d} s\right]+e^{-2 K \int_{0}^{t}\left\|Y_{m}(s, x)\right\|^{2} \mathrm{~d} s} \\
& \times\left\{-4 K \int_{0}^{t}\left(\left(Y_{m}(s, x), \eta_{m}^{h, x}(s)\right)\right) \mathrm{d} s \varphi\left(Y_{m}(t, x)\right)+\left(D \varphi\left(Y_{m}(t, x)\right), \eta_{m}^{h, x}(t)\right)\right\} \\
& \left.\times\left[\frac{1}{t} \int_{0}^{t}\left(Q_{m}^{-1 / 2} \eta_{m}^{h, x}(s), \mathrm{d} W(s)\right)+4 K \int_{0}^{t}\left(1-\frac{s}{t}\right)\left(\left(\eta_{m}^{h, x}(s), Y_{m}(s, x)\right)\right) \mathrm{d} s\right]\right)
\end{aligned}
$$

In $(3.1,3.2), Y_{m}, \eta_{m}^{h, x}$ and $\zeta_{m}^{h, x}$ are the solutions of $(2.22,2.27)$ and $(2.28)$ respectively. To estimate $D R_{t}^{m} \varphi$ and $D^{2} R_{t}^{m} \varphi$, we will use the following technical lemmas whose proofs are given in Appendix.

Lemma 3.1. (i) For any $k \in \mathbb{N}$, there exists $c_{3}(k)$ such that for any $t \in[0, T]$ and $x \in P_{m} H$

$$
\mathbb{E}\left[\left(\sup _{t \in[0, T]}\left|Y_{m}(t, x)\right|^{2}+\int_{0}^{T}\left\|Y_{m}(s, x)\right\|^{2} \mathrm{~d} s\right)^{k}\right] \leq c_{3}(k)\left(1+|x|^{2}\right)^{k}
$$

(ii) For any $\gamma \in(0,1 / 2)$ and any $k \in \mathbb{N}$ there exists $c_{4}(\gamma, k)$ and $c_{5}(\gamma, k)$ such that for $t \in[0, T]$ and $x \in P_{m} H$

$$
\mathbb{E}\left(e^{-c_{4}(\gamma, k) \int_{0}^{t}\left\|Y_{m}(s, x)\right\|^{2} \mathrm{~d} s}\left|Y_{m}(t, x)\right|_{\gamma}^{2 k}\right) \leq c_{5}(\gamma, k)\left(|x|_{\gamma}^{2}+1\right)^{k}
$$

Lemma 3.2. (i) For any $k \in \mathbb{N}$, there exists $c_{6}$ and $c_{7}(k)$ such that for any $t \in[0, T]$ and $x, h \in P_{m} H$

$$
\mathbb{E}\left[\left(e^{-c_{6} \int_{0}^{t}\left\|Y_{m}(s, x)\right\|^{2} \mathrm{~d} s}\left|\eta_{m}^{h, x}(t)\right|^{2}+\int_{0}^{t} e^{-c_{6} \int_{0}^{s}\left\|Y_{m}(\sigma, x)\right\|^{2} \mathrm{~d} \sigma}\left\|\eta_{m}^{h, x}(s)\right\|^{2} \mathrm{~d} s\right)^{k}\right] \leq c_{7}(k)|h|^{2 k}
$$

and

$$
\mathbb{E}\left[\left(e^{-c_{6} \int_{0}^{t}\left\|Y_{m}(s, x)\right\|^{2} \mathrm{~d} s}\left|\zeta_{m}^{h, x}(t)\right|^{2}+\int_{0}^{t} e^{-c_{6} \int_{0}^{s}\left\|Y_{m}(\sigma, x)\right\|^{2} \mathrm{~d} \sigma}\left\|\zeta_{m}^{h, x}(s)\right\|^{2} \mathrm{~d} s\right)^{k}\right] \leq c_{7}(k)|h|^{4 k}
$$

(ii) For any $\gamma \in(0,1 / 2)$ and $k \in \mathbb{N}$ there exists $c_{8}(\gamma)$ and $c_{9}(\gamma, k)$ such that for $t \in[0, T]$ and $x \in P_{m} H$ $\mathbb{E}\left[\left(e^{-c_{8}(\gamma) \int_{0}^{t}\left\|Y_{m}(s, x)\right\|^{2} \mathrm{~d} s}\left|\eta_{m}^{h, x}(t)\right|_{\gamma}^{2}+\int_{0}^{t} e^{-c_{8}(\gamma) \int_{0}^{s}\left\|Y_{m}(\sigma, x)\right\|^{2} \mathrm{~d} \sigma}\left|\eta_{m}^{h, x}(s)\right|_{\gamma+\frac{1}{2}}^{2} \mathrm{~d} s\right)^{k}\right] \leq c_{9}(\gamma, k)\left(|x|_{\gamma}^{2}+1\right)^{k}|h|_{\gamma}^{2 k}$, 


$$
\mathbb{E}\left[\left(e^{-c_{8}(\gamma) \int_{0}^{t}\left\|Y_{m}(s, x)\right\|^{2} \mathrm{~d} s}\left|\zeta_{m}^{h, x}(t)\right|_{\gamma}^{2}+\int_{0}^{t} e^{-c_{8}(\gamma) \int_{0}^{s}\left\|Y_{m}(\sigma, x)\right\|^{2} \mathrm{~d} \sigma}\left|\zeta_{m}^{h, x}(s)\right|_{\gamma+\frac{1}{2}}^{2} \mathrm{~d} s\right)^{k}\right] \leq c_{9}(\gamma, k)\left(|x|_{\gamma}^{2}+1\right)^{k}|h|_{\gamma}^{4 k} .
$$

With these lemmas, using $(3.1,3.2)$, we prove the following result.

Proposition 3.3. For any $\gamma_{2}>\gamma_{1}, \alpha \in[0,2], \beta \in[0,1]$ such that $\alpha+\beta \leq 2$, and $k \geq 0$, there exists $c_{10}\left(\gamma_{1}, \gamma_{2}, k\right)$ and $c_{11}\left(\gamma_{1}, \gamma_{2}, k\right)$ such that if $K \geq c_{10}\left(\gamma_{1}, \gamma_{2}, k\right)$ then for any $\varphi \in C_{b}\left(P_{m} H\right), t \in[0, T]$ :

$$
\left|R_{t}^{m} \varphi\right|_{\gamma_{2}, k+2 \alpha, \alpha+\beta} \leq c_{11}\left(\gamma_{1}, \gamma_{2}, k\right) t^{-\alpha\left(1-\left(\gamma_{1}-\gamma_{2}\right)\right)}|\varphi|_{\gamma_{2}, k, \beta} .
$$

Remark 1. It follows easily that $\left(R_{t}\right)$ satisfies the same smoothing properties. Also, we deduce that $\left(R_{t}\right)$ and $\left(R_{t}^{m}\right)$ can be extended to functions having polynomial growth.

Proof. In all the proof we set for simplicity:

$$
Y_{m}(s)=Y_{m}(s, x), \eta_{m}=\eta_{m}^{h, x}, \zeta_{m}=\zeta_{m}^{h, x} .
$$

We begin with $\alpha=\beta=0$ and write

$$
R_{t}^{m} \varphi(x)=\mathbb{E}\left(e^{-2 K \int_{0}^{t}\left\|Y_{m}\right\|^{2} \mathrm{~d} s} \varphi\left(Y_{m}(t)\right)\right)
$$

and

$$
\left|R_{t}^{m} \varphi(x)\right| \leq|\varphi|_{\gamma_{2}, k, 0} \mathbb{E}\left(e^{-2 K \int_{0}^{t}\left\|Y_{m}\right\|^{2} \mathrm{~d} s}\left(1+\left|Y_{m}(t)\right|_{\gamma_{2}}\right)^{k}\right) .
$$

The result follows in this case from Lemma 3.1.

We now consider the case $\alpha=1, \beta=0$. By (3.1) and the Cauchy-Schwartz inequality, we have:

$$
\left|\left(D R_{t}^{m} \varphi(x), h\right)\right| \leq|\varphi|_{\gamma_{2}, k, 0}\left[\mathbb{E}\left(e^{-2 K \int_{0}^{t}\left\|Y_{m}(s)\right\|^{2} \mathrm{~d} s}\left(1+\left|Y_{m}(t)\right|_{\gamma_{2}}\right)^{2 k}\right)\right]^{1 / 2}(A+B)^{1 / 2},
$$

where

$$
A=\frac{1}{t^{2}} \mathbb{E}\left[e^{-2 K \int_{0}^{t}\left\|Y_{m}(s)\right\|^{2} \mathrm{~d} s}\left(\int_{0}^{t}\left(Q_{m}^{-1 / 2} \eta_{m}(s), \mathrm{d} W(s)\right)\right)^{2}\right],
$$

and

$$
B=16 K^{2} \mathbb{E}\left[e^{-2 K \int_{0}^{t}\left\|Y_{m}(s)\right\|^{2} \mathrm{~d} s}\left(\int_{0}^{t}\left(1-\frac{s}{t}\right)\left(\left(\eta_{m}(s), Y_{m}(s)\right)\right) \mathrm{d} s\right)^{2}\right] .
$$

Using Lemma 3.1, $0 \leq 1-\frac{s}{t} \leq 1$, the Cauchy-Schwartz inequality and Lemma 3.2, we easily show that, for $K$ large enough,

$$
\mathbb{E}\left(e^{-2 K \int_{0}^{t}\left\|Y_{m}(s)\right\|^{2} \mathrm{~d} s}\left(1+\left|Y_{m}(t)\right|_{\gamma_{2}}\right)^{2 k}\right) \leq c_{12}(\gamma, k)\left(1+|x|_{\gamma_{2}}^{2}\right)^{2 k},
$$

and

$$
B \leq c_{13}|h|^{2} .
$$


To estimate $A$ we introduce

$$
z(t)=e^{-K \int_{0}^{t}\left\|Y_{m}(s)\right\|^{2} \mathrm{~d} s} \int_{0}^{t}\left(Q_{m}^{-1 / 2} \eta_{m}(s), \mathrm{d} W(s)\right),
$$

and use the Itô formula to obtain

$$
\begin{aligned}
\frac{1}{2} z^{2}(t)= & \int_{0}^{t}\left(-K z^{2}(s)\left\|Y_{m}\right\|^{2}+e^{-2 K \int_{0}^{s}\left\|Y_{m}(\sigma)\right\|^{2} \mathrm{~d} \sigma}\left|Q_{m}^{-1 / 2} \eta_{m}(s)\right|^{2}\right) \mathrm{d} s \\
& -\int_{0}^{t} z(s) e^{-K \int_{0}^{s}\left\|Y_{m}(\sigma)\right\|^{2} \mathrm{~d} \sigma}\left(Q_{m}^{-1 / 2} \eta_{m}(s), \mathrm{d} W(s)\right) .
\end{aligned}
$$

Thus

$$
A=\frac{1}{t^{2}} \mathbb{E}\left(z(t)^{2}\right) \leq \frac{1}{t^{2}} \mathbb{E}\left(\int_{0}^{t} e^{-2 K \int_{0}^{s}\left\|Y_{m}(\sigma)\right\|^{2} \mathrm{~d} \sigma}\left|Q_{m}^{-1 / 2} \eta_{m}(s)\right|^{2} \mathrm{~d} s\right) .
$$

We now use (2.13) and an interpolatory inequality in the space $D\left((-A)^{\gamma}\right)$ to derive

$$
\left|Q_{m}^{-1 / 2} \eta_{m}(s)\right|^{2} \leq c_{14}\left(\gamma_{1}, \gamma_{2}\right)\left|\eta_{m}(s)\right|_{\gamma_{2}}^{4\left(\gamma_{2}-\gamma_{1}\right)}\left|\eta_{m}(s)\right|_{\frac{1}{2}+\gamma_{2}}^{2-4\left(\gamma_{2}-\gamma_{1}\right)},
$$

and

$$
\begin{aligned}
A \leq & c_{14}\left(\gamma_{1}, \gamma_{2}\right) t^{-2} \mathbb{E}\left(\int_{0}^{t} e^{-K \int_{0}^{s}\left\|Y_{m}(\sigma)\right\|^{2} \mathrm{~d} \sigma}\left|\eta_{m}(s)\right|_{\gamma_{2}}^{4\left(\gamma_{2}-\gamma_{1}\right)}\left|\eta_{m}(s)\right|_{\frac{1}{2}+\gamma_{2}}^{2-4\left(\gamma_{2}-\gamma_{1}\right)} \mathrm{d} s\right) \\
\leq & c_{14}\left(\gamma_{1}, \gamma_{2}\right) t^{-2\left(1-\left(\gamma_{2}-\gamma_{1}\right)\right)} \sup _{t \in[0, T]}\left[\mathbb{E}\left(e^{-2 K \int_{0}^{t}\left\|Y_{m}(s)\right\|^{2} \mathrm{~d} s}\left|\eta_{m}(s)\right|_{\gamma_{2}}^{2}\right)\right]^{2\left(\gamma_{2}-\gamma_{1}\right)} \\
& \times\left[\mathbb{E}\left(\int_{0}^{T} e^{-2 K \int_{0}^{s}\left\|Y_{m}(\sigma)\right\|^{2} \mathrm{~d} \sigma}\left|\eta_{m}(s)\right|_{\frac{1}{2}+\gamma_{2}}^{2} \mathrm{~d} s\right)\right]^{1-2\left(\gamma_{2}-\gamma_{1}\right)}
\end{aligned}
$$

We now infer from Lemma 3.2 with $\gamma=\gamma_{2}$, that if $K$ is sufficiently large:

$$
A \leq c_{15}\left(\gamma_{1}, \gamma_{2}\right) t^{-2\left(1-\left(\gamma_{2}-\gamma_{1}\right)\right)}\left(1+|x|_{\gamma_{2}}^{2}\right)|h|_{\gamma_{2}}^{2} .
$$

Gathering (3.3-3.6) we deduce

$$
\left|\left(D R_{t}^{m} \varphi(x), h\right)\right| \leq c_{16}\left(\gamma_{1}, \gamma_{2}, k\right)|\varphi|_{\gamma_{2}, k, 0} t^{-\left(1-\left(\gamma_{1}-\gamma_{2}\right)\right)}\left(1+|x|_{\gamma_{2}}^{k+1}\right)|h|_{\gamma_{2}} .
$$

This gives the result for $\alpha=1$ and $\beta=0$. If $\alpha=\beta=1$, we use several times the Cauchy-Schwartz inequality to obtain from $(3.2)$

$$
\begin{aligned}
\left|\left(D^{2} R_{t}^{m} \varphi(x) h, h\right)\right| \leq & |\varphi|_{\gamma_{2}, k, 0}\left[\mathbb{E}\left(e^{-2 K \int_{0}^{t}\left\|Y_{m}(s)\right\|^{2} \mathrm{~d} s}\left(1+\left|Y_{m}(t)\right|_{\gamma_{2}}\right)^{2 k}\right)\right]^{1 / 2}(C+D)^{1 / 2} \\
& +\left[4 K | \varphi | _ { \gamma _ { 2 } , k , 0 } \left(\mathbb{E}\left(e^{-2 K \int_{0}^{t}\left\|Y_{m}(s)\right\|^{2} \mathrm{~d} s}\left(\int_{0}^{t}\left\|Y_{m}(s)\right\|^{2} \mathrm{~d} s\right)\right)^{4 / 8}\right.\right. \\
& \times\left(\mathbb{E}\left(e^{-2 K \int_{0}^{t}\left\|Y_{m}(s)\right\|^{2} \mathrm{~d} s}\left(\int_{0}^{t}\left\|\eta_{m}^{h}(s)\right\|^{2} \mathrm{~d} s\right)^{4}\right)\right)^{1 / 8} \\
& \left.+|\varphi|_{\gamma_{2}, k, 1}\left(\mathbb{E}\left(e^{-2 K \int_{0}^{t}\left\|Y_{m}(s)\right\|^{2} \mathrm{~d} s}\left|\eta_{m}^{h}(t)\right|_{\gamma_{2}}^{4}\right)\right)^{1 / 4}\right] \\
& \times\left(\mathbb{E}\left(e^{-2 K \int_{0}^{t}\left\|Y_{m}(s)\right\|^{2} \mathrm{~d} s}\left(1+\left|Y_{m}(t)\right|_{\gamma_{2}}^{4 k}\right)\right)^{1 / 4}(A+B)^{1 / 2},\right.
\end{aligned}
$$


where $A, B$ are as above,

$$
C=\frac{1}{t^{2}} \mathbb{E}\left(e^{-2 K \int_{0}^{t}\left\|Y_{m}(s)\right\|^{2} \mathrm{~d} s}\left(\int_{0}^{t}\left(Q_{m}^{-1 / 2} \zeta_{m}(s), \mathrm{d} W(s)\right)\right)^{2}\right),
$$

and

$$
D=16 K^{2} \mathbb{E}\left(e^{-2 K \int_{0}^{t}\left\|Y_{m}(s)\right\|^{2} \mathrm{~d} s}\left(\int_{0}^{t}\left(1-\frac{s}{t}\right)\left[\left(\left(\zeta_{m}^{h}(s), Y_{m}(s)\right)\right) \mathrm{d} s+\left\|\eta_{h}^{m}(s)\right\|^{2}\right] \mathrm{d} s\right)^{2}\right) .
$$

Similar arguments as in the preceding case show that if $K$ is sufficiently large, we have

$$
\left|\left(D^{2} R_{t}^{m} \varphi(x) h, h\right)\right| \leq c_{17}\left(\gamma_{1} \gamma_{2}, k\right)|\varphi|_{\gamma_{2}, k, 1} t^{-\left(1-\left(\gamma_{2}-\gamma_{1}\right)\right)}\left(1+|x|_{\gamma_{2}}\right)^{k+2}|h|_{\gamma_{2}}^{2},
$$

and we obtain the case $\alpha=\beta=1$.

Using the semigroup property, we have

$$
R_{t}^{m} \varphi=R_{t / 2}^{m}\left(R_{t / 2}^{m} \varphi\right)
$$

and using the two preceding results we have

$$
\left|R_{t}^{m} \varphi\right|_{\gamma_{2}, k+3,2} \leq c_{18}\left(\gamma_{1}, \gamma_{2}, k\right)\left|R_{t / 2}^{m} \varphi\right|_{\gamma_{2}, k+1,1}(t / 2)^{-\left(1-\left(\gamma_{2}-\gamma_{1}\right)\right)} \leq c_{19}\left(\gamma_{1}, \gamma_{2}, k\right)(t / 2)^{-2\left(1-\left(\gamma_{2}-\gamma_{1}\right)\right)}|\varphi|_{\gamma_{2}, k, 0},
$$

so that the result is true for $\alpha=2, \beta=0$. The other cases are proved by interpolation.

\section{A PRIORI ESTIMATES AND CONSTRUCTION OF A SOLUtion}

We now use Proposition 3.3 to estimate the solution $v^{m}$ of (2.23) and also $u^{m}$. We first estimate the first and the last term of (2.23).

Lemma 4.1. There exists $c_{20}$ such that, if $K$ is large enough, for any $m \in \mathbb{N}$ and $t \in[0, T]$ :

$$
\left|R_{t}^{m} \widetilde{\varphi}+\int_{0}^{t} R_{t-s}^{m} \widetilde{g} \mathrm{~d} s\right|_{0,0,2} \leq c_{20} .
$$

Proof. For $x \in P_{m} H, t \in[0, T]$, we have

$$
\left|R_{t}^{m} \widetilde{\varphi}(x)\right|=\mathbb{E}\left(e^{-2 K \int_{0}^{t}\left\|Y_{m}(s, x)\right\|^{2} \mathrm{~d} s-K\left|Y_{m}(t, x)\right|^{2}}\left|Y_{m}(t, x)\right|^{2}\right) \leq \frac{K}{e} .
$$

Moreover for $h \in P_{m} H$

$$
\begin{aligned}
\left(D R_{t}^{m} \widetilde{\varphi}(x), h\right)= & \mathbb{E}\left[e ^ { - 2 K \int _ { 0 } ^ { t } \| Y _ { m } ( s , x ) \| ^ { 2 } \mathrm { d } s - K | Y _ { m } ( t , x ) | ^ { 2 } } \left(-4 K \int_{0}^{t}\left(\left(Y_{m}(s, x), \eta_{m}^{h, x}(s)\right)\right) \mathrm{d} s\right.\right. \\
& \left.\left.-2 K\left(Y_{m}(t, x), \eta_{m}^{h, x}(t)\right)\left|Y_{m}(t, x)\right|^{2}+2\left(Y_{m}(t, x), \eta_{m}^{h, x}(t)\right)\right)\right],
\end{aligned}
$$

and we obtain from elementary inequalities and Lemma 3.2:

$$
\left|\left(D R_{t}^{m} \widetilde{\varphi}(x), h\right)\right| \leq c_{21}|h| .
$$


The second differential of $R_{t}^{m} \widetilde{\varphi}$ is estimated in the same way. Also

$$
\int_{0}^{t} R_{t-s}^{m} \widetilde{g}(x) \mathrm{d} s=\mathbb{E}\left(\int_{0}^{t} e^{-2 K \int_{0}^{t-s}\left\|Y_{m}(\sigma, x)\right\|^{2} \mathrm{~d} \sigma-K\left|Y_{m}(t-s, x)\right|^{2}}\left|\operatorname{rot} Y_{m}(t-s, x)\right|^{2} \mathrm{~d} s\right)
$$

and again this term and its derivatives are estimated by elementary inequalities and Lemma 3.2.

We will use the following simple lemma whose proof is left to the reader and uses $(2.7,2.18)$.

Lemma 4.2. Let $\gamma \leq \tilde{\gamma}_{1}, k \geq \alpha$. There exists $c_{21}(\gamma, k, \alpha)$ such that for any $v \in C_{\gamma, k, 1+\alpha}, \tilde{F}\left(x, v, v_{x}\right)$ belongs to $C_{\gamma, k+2, \alpha}$ and

$$
\left|\tilde{F}\left(x, v, v_{x}\right)\right|_{\gamma, k+2, \alpha} \leq c_{21}(\gamma, k, \alpha)\left(1+|v|_{\gamma, k, 1+\alpha}\right) .
$$

We now give the estimate on $v^{m}$.

Proposition 4.3. For any $\gamma_{1}<\gamma \leq \tilde{\gamma}_{1}$, there exists $d\left(\gamma_{1}, \gamma\right)$ and $c_{22}\left(\gamma_{1}, \gamma\right)$ such that for any $m \in \mathbb{N}$ :

$$
\sup _{t \in[0, T]}\left|v^{m}(t, \cdot)\right|_{\gamma, d\left(\gamma_{1}, \gamma\right), 2} \leq c_{22}\left(\gamma_{1}, \gamma\right) .
$$

Proof. We first recall that $u^{m}$ is the value function associated to the approximated control problem. We deduce

$$
0 \leq u^{m}(T, x)=\min _{z \in \mathcal{M}_{R} \cap L_{W}^{2}\left(\Omega \times[0, T] ; P_{m} H\right)} J_{m}(z) \leq J_{m}(0) \leq c_{23}\left(1+|x|^{2}\right) .
$$

We could have considered the same control problem on $[t, T]$ and obtained

$$
0 \leq u^{m}(t, x) \leq c_{23}\left(1+|x|^{2}\right)
$$

This gives

$$
\left|v^{m}(t, x)\right| \leq c_{23}\left(1+|x|^{2}\right) e^{-K|x|^{2}}
$$

for any $m \in \mathbb{N}, t \in[0, T], x \in P_{m} H$. We deduce

$$
\left|v^{m}(t, \cdot)\right|_{0, k, 0} \leq c_{24}(k)
$$

for any $k \in \mathbb{N}$.

We set $\varepsilon=\gamma-\gamma_{1}>0$. Let us choose $\alpha>0$ such that:

$$
(1+\alpha)(1-\varepsilon)<1 .
$$

Then by (2.23)

$$
\begin{aligned}
\left|v^{m}(t, \cdot)\right|_{\gamma, d_{\alpha}+4+2 \alpha, 1+\alpha} \leq & \left|R_{t}^{m} \tilde{\varphi}(\cdot)+\int_{0}^{t} R_{t-s}^{m} \tilde{g}(\cdot) \mathrm{d} s\right|_{\gamma, d_{\alpha}+4+2 \alpha, 1+\alpha} \\
& +\int_{0}^{t}\left|R_{t-s}^{m} \tilde{F}_{m}\left(\cdot, v^{m}(s, \cdot), v_{x}^{m}(s, \cdot)\right)\right|_{\gamma, d_{\alpha}+4+2 \alpha, 1+\alpha} \mathrm{d} s
\end{aligned}
$$


where we have set $d_{\alpha}=\frac{1}{\alpha}(4+2 \alpha)$. We use Proposition 3.3 and Lemma 4.2 to derive

$$
\begin{aligned}
& \int_{0}^{t}\left|R_{t-s}^{m} \tilde{F}_{m}\left(\cdot, v^{m}(s, \cdot), v_{x}^{m}(s, \cdot)\right)\right|_{\gamma, d_{\alpha}+4+2 \alpha, 1+\alpha} \mathrm{d} s \\
& \leq c_{23}\left(\gamma_{1}, \gamma, d_{\alpha}+2\right) \int_{0}^{t}(t-s)^{-(1+\alpha)(1-\varepsilon)}\left|\tilde{F}_{m}\left(\cdot, v^{m}(s, \cdot), v_{x}^{m}(s, \cdot)\right)\right|_{\gamma, d_{\alpha}+2,0} \mathrm{~d} s \\
& \leq c_{25}\left(\gamma_{1}, \gamma, \alpha\right) \int_{0}^{t}(t-s)^{-(1+\alpha)(1-\varepsilon)}\left(\left|v^{m}(s, \cdot)\right|_{\gamma, d_{\alpha}, 1}+1\right) \mathrm{d} s \\
& \leq c_{26}\left(\gamma_{1}, \gamma, \alpha\right)\left(\sup _{t \in[0, T]}\left|v^{m}(t, \cdot)\right|_{\gamma, d_{\alpha}, 1}+1\right) \mathrm{d} s .
\end{aligned}
$$

By interpolation we have

$$
\left|v^{m}(t, \cdot)\right|_{\gamma, d_{\alpha}, 1} \leq c_{27}(\gamma, \alpha)\left|v^{m}(t, \cdot)\right|_{\gamma, 0,0}^{\frac{1}{1+\alpha}}\left|v^{m}(t, \cdot)\right|_{\gamma, d_{\alpha}+4+2 \alpha, 1+\alpha}^{\frac{\alpha}{1+\alpha}},
$$

and, by (4.1), Lemma 3.1, (4.2), (4.3), we deduce

$$
\sup _{t \in[0, T]}\left|v^{m}(t, \cdot)\right|_{\gamma, d_{\alpha}+4+2 \alpha, 1+\alpha} \leq c_{28}\left(\gamma_{1}, \gamma, \alpha\right) .
$$

It is now easy to use this estimate and to derive from Proposition 3.3 (with $(\alpha, \beta)$ replaced by $(1+\alpha, \alpha)$ and Lemma 4.1, 4.2 an estimate on $v^{m}(t, \cdot)$ in $C^{\gamma, 8+4 \alpha, 1+2 \alpha}$. After a finite number of steps of this argument, we obtain

$$
\sup _{t \in[0, T]}\left|v^{m}(t, \cdot)\right|_{\gamma, \widetilde{d}_{\alpha}, 2} \leq c_{29}(\gamma, \alpha)
$$

We deduce easily from Proposition 4.3 and (2.21) that for any $r>0$

$$
\sup _{t \in[0, T], x \in D(A),|A x| \leq r}\left|D_{t} v^{m}(t, x)\right| \leq c_{30}(r),
$$

where $c_{30}(r)$ depends only on $r$.

Then, arguing as in [8], Section 5, we prove that there exists a subsequence $\left(v^{m_{k}}\right)$ and a function $v$ in $C^{\gamma, d\left(\gamma_{1} \gamma\right), 2}$ for any $\gamma \in\left(\gamma, \tilde{\gamma_{1}}\right)$ such that

$$
\begin{gathered}
v^{m_{k}}(t, x) \rightarrow v(t, x) \text { in } \mathbb{R}, \\
D v^{m_{k}}(t, x) \rightarrow D v(t, x) \text { in } D\left((-A)^{-\gamma}\right),
\end{gathered}
$$

for any $\gamma \in\left(\gamma, \tilde{\gamma_{1}}\right), x \in D\left((-A)^{\gamma}\right), t \in[0, T]$. Moreover for any $r>0$ :

$$
|D v(t, x)-D v(t, y)|_{-\gamma} \leq c_{31}(r)|x-y|_{\gamma},
$$

for $x, y \in D\left((-A)^{\gamma}\right)$ such that $|x|_{\gamma},|y|_{\gamma} \leq r$. Then, using (4.4), (4.5), (4.6) and standard results on the convergence of the Galerkin approximation $Y_{m}$ to the solution $Y$ of (2.11) we get that $v$ satisfies (2.17), from which we deduce that $v$ is continuous in time:

$$
v \in C\left([0, T], C^{\gamma, d, 2}\right)
$$

for any $\gamma \in\left(\gamma_{1}, \tilde{\gamma_{1}}\right)$ and some $d \geq 0$. 


\section{A. Proof of Lemmas 3.1 and 3.2}

Proof of Lemma 3.1 - (i) is standard and follows easily from the Itô formula and the martingale inequalities.

To prove (ii) we set

$$
w_{m}(t)=\int_{0}^{t} e^{(t-s) A} Q_{m}^{1 / 2} \mathrm{~d} W(s), y_{m}=Y_{m}-w_{m}
$$

then

$$
\frac{\mathrm{d} y_{m}}{\mathrm{~d} t}=A y_{m}+b_{m}\left(Y_{m}\right)
$$

and taking the scalar product with $(-A)^{2 \gamma} y_{m}$ yields

$$
\begin{aligned}
\frac{1}{2} \frac{\mathrm{d}}{\mathrm{d} t}\left|y_{m}\right|_{\gamma}^{2}+\left|y_{m}\right|_{\gamma+\frac{1}{2}}^{2} & =\left(b_{m}\left(Y_{m}\right),(-A)^{2 \gamma} y_{m}\right) \\
& \leq c_{1}(\gamma)\left|Y_{m}\right|_{\gamma}\left\|Y_{m}\right\|\left|y_{m}\right|_{\frac{1}{2}-\gamma} \leq c_{1}(\gamma)^{2}\left\|Y_{m}\right\|^{2}\left|y_{m}\right|_{\gamma}^{2}+c_{1}(\gamma)^{2}\left\|Y_{m}\right\|^{2}\left|w_{m}\right|_{\gamma}^{2}+\frac{1}{2}\left|y_{m}\right|_{1 / 2-\gamma}^{2}
\end{aligned}
$$

by (2.3) and elementary inequalities. Thus, by the Gronwall lemma:

$$
e^{-2 c_{1}(\gamma)^{2} \int_{0}^{t}\left\|Y_{m}(s, x)\right\|^{2} \mathrm{~d} s}\left|y_{m}\right|_{\gamma}^{2} \leq|x|_{\gamma}^{2}+\sup _{t \in[0, T]}\left|w_{m}\right|_{\gamma}^{2}
$$

The conclusion follows easily since, as it is well known:

$$
\mathbb{E}\left(\sup _{t \in[0, T]}\left|w_{m}\right|_{\gamma}^{2 k}\right) \leq C_{1}(k)
$$

for any $k \in \mathbb{N}$.

Proof of Lemma 3.2 - Since no ambiguity is possible here we set for simplicity $\eta_{m}^{h, x}=\eta_{m}$.

We take the scalar product of (2.27) with $\eta_{m}$ and use (2.1) to get

$$
\frac{1}{2} \frac{\mathrm{d}}{\mathrm{d} t}\left|\eta_{m}\right|^{2}+\left\|\eta_{m}\right\|^{2}=b_{m}\left(\eta_{m}, Y_{m}, \eta_{m}\right) \leq c_{1}\left|\eta_{m}\right|_{1 / 4}^{2}\left\|Y_{m}\right\| \leq c_{2}\left|\eta_{m}\right|\left\|\eta_{m}\right\|\left\|Y_{m}\right\| \leq \frac{1}{2}\left\|\eta_{m}\right\|^{2}+\frac{1}{2} c_{2}^{2}\left\|Y_{m}\right\|^{2}\left|\eta_{m}\right|^{2}
$$

by (2.3) and an interpolatory inequality. The first claim of (i) follows. Similarly, using (2.2) we have for $\zeta_{m}=\zeta_{m}^{h, x}$

$$
\frac{1}{2} \frac{\mathrm{d}}{\mathrm{d} t}\left|\zeta_{m}\right|^{2}+\left\|\zeta_{m}\right\|^{2} \leq c_{3}\left|\zeta_{m}\right|^{2}\left\|Y_{m}\right\|^{2}+c_{4}\left|\eta_{m}\right|^{2}\left\|\eta_{m}\right\|^{2}+\frac{1}{2}\left\|\zeta_{m}\right\|^{2}
$$

Again we can conclude easily using the above inequality and noticing that it can be assumed that $c_{3} \geq c_{2}^{2}$.

We take the scalar product of $(2.27)$ with $(-A)^{2 \gamma} \eta_{m}$ and use again (2.3) to obtain

$$
\frac{1}{2} \frac{\mathrm{d}}{\mathrm{d} t}\left|\eta_{m}\right|_{\gamma}^{2}+\left|\eta_{m}\right|_{\gamma+1 / 2}^{2} \leq c_{1}(\gamma)\left|Y_{m}\right|_{\gamma}\left\|\eta_{m}\right\|\left|\eta_{m}\right|_{1 / 2+\gamma}+c_{1}(\gamma)\left|\eta_{m}\right|_{\gamma}\left\|Y_{m}\right\|\left|\eta_{m}\right|_{1 / 2+\gamma}
$$

from which we have after few manipulations

$$
\frac{\mathrm{d}}{\mathrm{d} t}\left|\eta_{m}\right|_{\gamma}^{2}+\left|\eta_{m}\right|_{\gamma+1 / 2}^{2} \leq c_{4}(\gamma)\left|Y_{m}\right|_{\gamma}^{2}\left\|\eta_{m}\right\|^{2}+c_{4}(\gamma)\left|\eta_{m}\right|_{\gamma}^{2}\left\|Y_{m}\right\|^{2}
$$


and

$$
e^{-c_{4}(\gamma) \int_{0}^{t}\left\|Y_{m}(s)\right\|^{2} \mathrm{~d} s}\left|\eta_{m}\right|_{\gamma}^{2}+\int_{0}^{t} e^{-c_{4}(\gamma) \int_{0}^{s}\left\|Y_{m}(\sigma)\right\|^{2} \mathrm{~d} \sigma}|\eta|_{\gamma+1 / 2}^{2} \mathrm{~d} s \leq|h|_{\gamma}^{2}+\int_{0}^{t} e^{-c_{4}(\gamma) \int_{0}^{s}\left\|Y_{m}(\sigma)\right\|^{2} \mathrm{~d} \sigma}\left|Y_{m}\right|_{\gamma}^{2}\left\|\eta_{m}\right\|^{2} \mathrm{~d} s
$$

We conclude thanks to Lemma 3.1 and the first part of the lemma. The final inequality concerning $\zeta_{m}^{h}$ is proved similarly.

\section{REFERENCES}

[1] F. Abergel and R. Temam, On some control problems in fluid mechanics. Theor. and Comp. Fluid Dynamics 1 (1990) 303-325.

[2] V. Barbu and S. Sritharan, $H^{\infty}$-control theory of fluids dynamics. Proc. R. Soc. Lond. A 454 (1998) 3009-3033.

[3] T. Bewley, P. Moin and R. Temam, Optimal and robust approaches for linear and nonlinear regulartion problems in fluid mechanics, AIAA 97-1872, 28th AIAA Fluid Dynamics Conference and 4th AIAA Shear Flow Control Conference (1997).

[4] P. Cannarsa and G. da Prato, Some results on nonlinear optimal control problems and Hamilton-Jacobi equations in infinite dimensions. J. Funct. Anal. 90 (1990) 27-47.

[5] P. Cannarsa and G. da Prato, Direct solution of a second order Hamilton-Jacobi equation in Hilbert spaces, in: Stochastic partial differential equations and applications, G. da Prato and L. Tubaro Eds, Pitman Research Notes in Mathematics Series n.268 (1992) pp. 72-85.

[6] S. Cerrai, Optimal control problem for stochastic reaction-diffusion systems with non Lipschitz coefficients (to appear).

[7] H. Choi, R. Temam, P. Moin and J. Kim, Feedback control for unsteady flow and its application to the stochastic Burgers equation. J. Fluid Mech. 253 (1993) 509-543.

[8] G. da Prato and A. Debussche, Differentiability of the transition semigroup of stochastic Burgers equation. Rend. Acc. Naz. Lincei, s.9, v. 9 (1998) 267-277.

[9] G. da Prato and A. Debussche, Dynamic Programming for the stochastic Burgers equations. Annali di Mat. Pura ed Appl. (to appear).

[10] G. da prato and J. Zabczyk, Differentiability of the Feynman-Kac semigroup and a control application. Rend. Mat. Acc. Lincei. s.9, v. 8 (1997) 183-188.

[11] H. Fattorini and S. Sritharan, Existence of optimal controls for viscous flow problems. Proc. R. Soc. Lond. A 439 (1992) $81-102$.

[12] F. Gozzi, Regularity of solutions of a second order Hamilton-Jacobi equation and application to a control problem. Commun. in partial differential equations 20 (1995) 775-826.

[13] F. Gozzi, Global Regular Solutions of Second Order Hamilton-Jacobi Equations in Hilbert spaces with locally Lipschitz nonlinearities. J. Math. Anal. Appl. 198 (1996) 399-443.

[14] P.L. Lions, Viscosity solutions of fully nonlinear second-order equations and optimal stochastic control in infinite dimensions. Part I: The case of bounded stochastic evolution. Acta Math. 161 (1988) 243-278. Part II: Optimal control of Zakai's equation, in Stochastic partial differential equations and applications, G. da Prato and L. Tubaro Eds, Lecture Notes in Mathematics No. 1390, Springer-Verlag (1990) 147-170. Part III: Uniqueness of viscosity solutions for general second order equations. $J$. Funct. Anal. 86 (1991) 1-18.

[15] S. Sritharan, Dynamic programming of the Navier-Stokes equations. Syst. Cont. Lett. 16 (1991) 299-307.

[16] S. Sritharan, An introduction to deterministic and stochastic control of viscous flow, in Optimal control of viscous flows, p. 1-42, SIAM, Philadelphia, S. Sritharan Ed.

[17] A. Swiech, Viscosity solutions of fully nonlinear partial differential equations with "unbounded" terms in infinite dimensions, Ph.D. thesis, University of California at Santa Barbara (1993).

[18] R. Temam, T. Bewley and P.Moin, Control of turbulent flows, Proc. of the 18th IFIP TC7, Conf. on system modelling ond optimization, Detroit, Michigan (1997).

[19] R. Temam, The Navier-Stokes equation, North-Holland (1977). 